\title{
Mitigating the Agglomeration of Nanofiller in a Mixed Matrix Membrane by Incorporating an Interface Agent
}

\author{
Manh-Tuan Vu ${ }^{1,2}$, Gloria M. Monsalve-Bravo ${ }^{1}{ }^{1}$, Rijia Lin ${ }^{1, *(\mathbb{D}}$, Mengran Li ${ }^{1}$, Suresh K. Bhatia ${ }^{1}(\mathbb{D}$ \\ and Simon Smart 1,3,* \\ 1 School of Chemical Engineering, The University of Queensland, Brisbane, QLD 4072, Australia; \\ manh.vu@uq.net.au (M.-T.V.); g.monsalvebravo@uq.edu.au (G.M.M.-B.); m.li6@uq.edu.au (M.L.); \\ s.bhatia@uq.edu.au (S.K.B.) \\ 2 Institute for Tropical Technology, Vietnam Academy of Science and Technology, Hanoi 100000, Vietnam \\ 3 Dow Centre for Sustainable Engineering Innovation, The University of Queensland, \\ Brisbane, QLD 4072, Australia \\ * Correspondence: r.lin1@uq.edu.au (R.L.); s.smart@uq.edu.au (S.S.)
}

check for updates

Citation: Vu, M.-T.; Monsalve-Bravo, G.M.; Lin, R.; Li, M.; Bhatia, S.K.; Smart, S. Mitigating the Agglomeration of Nanofiller in a Mixed Matrix Membrane by Incorporating an Interface Agent. Membranes 2021, 11, 328. https:// doi.org/10.3390/membranes11050328

Academic Editor: Clara

Casado Coterillo

Received: 25 March 2021

Accepted: 25 April 2021

Published: 29 April 2021

Publisher's Note: MDPI stays neutral with regard to jurisdictional claims in published maps and institutional affiliations.

Copyright: (c) 2021 by the authors. Licensee MDPI, Basel, Switzerland. This article is an open access article distributed under the terms and conditions of the Creative Commons Attribution (CC BY) license (https:/ / creativecommons.org/licenses/by/ $4.0 /)$.

\begin{abstract}
Nanodiamonds (ND) have recently emerged as excellent candidates for various applications including membrane technology due to their nanoscale size, non-toxic nature, excellent mechanical and thermal properties, high surface areas and tuneable surface structures with functional groups. However, their non-porous structure and strong tendency to aggregate are hindering their potential in gas separation membrane applications. To overcome those issues, this study proposes an efficient approach by decorating the ND surface with polyethyleneimine (PEI) before embedding it into the polymer matrix to fabricate $M M M s$ for $\mathrm{CO}_{2} / \mathrm{N}_{2}$ separation. Acting as both interfacial binder and gas carrier agent, the PEI layer enhances the polymer/filler interfacial interaction, minimising the agglomeration of ND in the polymer matrix, which is evidenced by the focus ion beam scanning electron microscopy (FIB-SEM). The incorporation of PEI into the membrane matrix effectively improves the $\mathrm{CO}_{2} / \mathrm{N}_{2}$ selectivity compared to the pristine polymer membranes. The improvement in $\mathrm{CO}_{2} / \mathrm{N}_{2}$ selectivity is also modelled by calculating the interfacial permeabilities with the Felske model using the gas permeabilities in the MMM. This study proposes a simple and effective modification method to address both the interface and gas selectivity in the application of nanoscale and non-porous fillers in gas separation membranes.
\end{abstract}

Keywords: nanodiamond; mixed matrix membrane; filler dispersion; Felske model; gas separation

\section{Introduction}

Membranes are recognised as an energy-efficient and environmental-friendly separation technology for gas separation. However, most of the conventional polymer membranes are restricted to a trade-off between mass transport rates and separation efficiency, while inorganic membranes face the limit of poor scalability and high cost [1,2]. Mixed matrix membranes (MMMs), which can be prepared by embedding selective filler materials into a continuous polymer matrix, offer an opportunity to overcome the drawbacks associated with pure polymer or inorganic membranes [3-6]. With proper selection of the filler/polymer pair, mixed matrix membranes benefit from both the ease of processing of the polymer materials and superior separation properties of the filler particles. The fabrication of MMMs is analogous to that of pure polymer membranes, which means that MMMs could be manufactured on industrial scales by modifying existing polymer membrane production technologies [7].

Pair-wise selection of the filler and polymer is the basis for the MMM fabrication because it is relevant to the morphology and performance of the membrane. The selection of polymer determines the minimum gas transport performance (diffusivity and solubility) of the membrane. The embedded filler defines the improvement of the gas permeability and 
selectivity of the MMM [7]. Generally, nano sized fillers are preferred due to their relatively good dispersion in the polymer matrix and the strong interaction with the polymer phase.

Nanodiamonds (NDs) are specific carbon nano-crystalline particles with a tetrahedral diamond structure [8]. ND particles have primarily a spherical shape with an average diameter of $5-10 \mathrm{~nm}$ with a narrow particle size distribution. NDs are potential candidates for various applications, such as composite materials $[9,10]$, quantum sensing and imaging [11,12], and biomedical applications [13,14], owing to NDs' chemical stability, thermal conductivity, biocompatibility, hardness, and photoluminescence properties [15-17].

Recently, NDs have been applied as the nanofiller into several polymer matrices for the fabrication of polymer composites. The incorporation of ND can improve the strength, thermal stability and conductivity, electrical conductivity, chemical resistance and biocompatibility of the polymer matrices $[18,19]$. Functionalised ND was embedded into epoxy by in situ polymerization. The incorporation of the ND resulted in high thermal stability and thermal conductivity of the epoxy composite compared to the pure polymer matrix [20]. Etemadi et al. introduced amino-functionalised ND into cellulose acetate membrane for anti-biofouling in membrane bioreactor. The incorporation of amino-functionalised ND improves the hydrophilicity of the membrane [21]. Carboxylated ND was prepared by $\mathrm{Li}$ et al. and embedded in polyvinylidene fluoride. The polyvinylidene fluoride membrane blended with carboxylated ND showed higher hydrophilicity and water permeability, as well as improved anti-fouling performance [22]. A composite membrane based on polyvinylidene fluoride/nanodiamond has been synthesised for better desalination performance [23]. Nanodiamond was firstly introduced in an interfacial polymerisation system for the fabrication of nanofiltration membranes [24]. The introduction of ND transforms the membrane structure from nodules to ridges at the nanoscale. The resulted nanofiltration membrane presented a high-water permeability and salt rejection. Other work by Polotskaya et al. selected ND as an inorganic filler for poly(2,6-dimethyl-1,4-phenylene oxide) membranes for gas separation [25]. The same group also incorporated ND into polyphenylene-isophthalamide matrix to prepare MMMs for gas separation [26]. Their results showed that the selectivity of $\mathrm{H}_{2} / \mathrm{N}_{2}$ and $\mathrm{O}_{2} / \mathrm{N}_{2}$ gas pairs increased with the $\mathrm{ND}$ concentration up to $3 \mathrm{wt} . \%$.

The dispersion of filler particles in the continuous polymer phase is vital to the performance of the MMMs. However, NDs tend to form aggregates spontaneously to minimise surface energy [24]. The particle agglomeration can lead to the formation of non-selective interfacial voids inside the particle aggregates, which can cause the decline of membrane gas selectivity. The most common approach to improve filler dispersion is the priming method. In this approach, the filler particles are coated with a thin layer of the polymer by introducing a small amount of polymer into the filler suspension, before mixing it with the remaining bulk of the polymer. The priming method can decrease stress at the filler/polymer interface, minimising the aggregation of the fillers [27]. Nevertheless, applying the priming process by itself will not eliminate the formation of particle agglomeration in MMMs with nanoparticles. The dispersion of inorganic fillers can also be improved by applying the interfacial polymerisation processes [28]. In this approach, the inorganic filler is dispersed along with the organic monomer and the polymerisation will occur on the interface between filler and monomer.

In this study, polyether block amide Pebax (MH 1657) was chosen as the polymer matrix for the MMM fabrication due to its excellent $\mathrm{CO}_{2}$ selectivity [29]. Pebax consists of polyamide (PA) as hard segments and polyether (PE) as soft segments in the polymer chains, where the hard crystalline PA block provides mechanical strength and the soft polyether block plays as the gas permeable phase due to its high chain mobility [30]. Nanodiamond was chosen as the filler for MMM fabrication. We propose here an effective and convenient route for mitigating the agglomeration of NDs in MMM by incorporating a third interface agent. Controllable decoration of low molecular weight polyethyleneimine (PEI) on NDs was performed before embedding into the polymer matrix. PEI acts as a wetting and binding material between $\mathrm{ND}$ and polymer, as well as a $\mathrm{CO}_{2}$ carrier agent. 
Focused ion beam scanning electron microscopy (FIB-SEM) was applied to provide a quantitative analysis of the distribution of ND aggregates within the polymer matrix. Improved dispersion of NDs has been achieved with the incorporation of PEI and the size and quantity of the ND aggregates are significantly reduced. The $\mathrm{CO}_{2} / \mathrm{N}_{2}$ selectivity of Pebax/ND-PEI MMMs have been improved compared to the Pebax/ND MMMs and pristine Pebax membranes. Finally, such an enhancement of the polymer/filler interfacial properties is corroborated by calculating the interface thickness and gas permeability with the Felske model [31-34] using the $\mathrm{CO}_{2} / \mathrm{N}_{2}$ Pebax/ND-PEI MMM permeabilities.

\section{Materials and Methods}

\subsection{Materials}

Nanodiamond ( $\geq 97 \%$ trace metals basis, particle size $<10 \mathrm{~nm}$ ) and polyethyleneimine (PEI, average $M_{n} \sim 1200$, average $M_{w} \sim 1300$ ) were purchased from Sigma-Aldrich. The received nanodiamond powder was heated at $425^{\circ} \mathrm{C}$ in the air in a furnace for $4 \mathrm{~h}$ (heating rate $10^{\circ} \mathrm{C} / \mathrm{min}$ ) to maximise the oxygen functional groups on the surface of ND [35]. Pebax (MH 1657) was kindly supplied by Arkema, France. Ethanol was obtained from Merck. All the chemicals were used without further purification. Cylinders of pure $\mathrm{CO}_{2}$ and $\mathrm{N}_{2}$ were supplied by Coregas.

\subsection{Nanodiamond Surface Modification}

An amount of $2 \mathrm{~g}$ of polyethyleneimine (PEI) was dissolved in $15 \mathrm{~mL}$ of deionised water and stirred in $30 \mathrm{~min}$ to form a homogeneous solution. $0.2 \mathrm{~g}$ of ND was then dispersed into the PEI contained solution and sonicated for $15 \mathrm{~min}$ in order to completely dispersed the ND nanoparticles. The well-dispersed mixture was subsequently heated to $70{ }^{\circ} \mathrm{C}$ whilst stirring at $250 \mathrm{rpm}$ for $24 \mathrm{~h}$. The mixture was then centrifuged and washed with deionised water for at least three times before drying in a vacuum oven at $100{ }^{\circ} \mathrm{C}$ for $24 \mathrm{~h}$ and stored under vacuum before use. The obtained sample was then labelled as ND-PEI.

\subsection{Fabrication of Nanodiamond Incorporated Mixed Matrix Membranes}

For the neat Pebax membrane, $0.48 \mathrm{~g}$ of Pebax was dissolved in a mixture of ethanol/water (70 wt. \%/30 wt. \%) by heating up to $70{ }^{\circ} \mathrm{C}$ and stirring for $6 \mathrm{~h}$. The resulting solution was then cast on a flat glass surface at $30 \mu \mathrm{m}$ of thickness and dried in a vacuum oven at $100{ }^{\circ} \mathrm{C}$ in $24 \mathrm{~h}$. The obtained membrane was then peeled off the glass plate and stored under vacuum before use.

For the mixed matrix membrane fabrication, a quantity of nanodiamond (ND or ND-PEI) was dispersed into a mixed solution of ethanol/water (70 wt. $\% / 30 \mathrm{wt} . \%$ ) and sonicated for $15 \mathrm{~min}$. Pebax was then slowly added into the mixture while heating to $70{ }^{\circ} \mathrm{C}$ along with sonication several times during the process. The amount of Pebax and nanodiamond was calculated in order to form the ratio: Pebax/ND = 99.9/0.1, 99.5/0.5, $99 / 1,98.5 / 1.5$, referred to as the nominal ND loading in Section 3. The resulting mixture was cast onto a clean glass plate at a thickness of $30 \mu \mathrm{m}$ and dried at $100{ }^{\circ} \mathrm{C}$ in $24 \mathrm{~h}$ before peeling off. The thickness of pure Pebax and MMMs were measured using a micrometre within the range of $40-50 \mu \mathrm{m}$. The membranes were stored with desiccant under vacuum before gas permeation tests and characterisation.

\subsection{Characterisation}

The content of carbon, hydrogen and nitrogen in ND and ND-PEI samples was quantified by a Thermo Scientific ${ }^{\text {TM }}$ FLASH 2000 CHNS/O Analyzer. X-ray photoelectron spectrometer (XPS) was applied by a Kratos Axis Ultra XPS equipped with a $165 \mathrm{~mm}$ hemispherical electron energy analyser and a monochromatic $\mathrm{Al} \mathrm{K \alpha}(1486.6 \mathrm{eV})$ radiation at $150 \mathrm{~W}(15 \mathrm{kV}, 10 \mathrm{~mA})$. The $\mathrm{C} 1 \mathrm{~s}$ main peak position was fixed at $284.8 \mathrm{eV}$ and taken as an internal standard. The loading of PEI on the ND particles was calculated by the Perkin Elmer Instruments STA 6000 Thermo Gravimetric Analyser. The ND-PEI sample 
was heated under an air atmosphere at a uniform heating rate of $5^{\circ} \mathrm{C} \mathrm{min}^{-1}$ from 40 to $800^{\circ} \mathrm{C}$. Fourier-transform infrared (FTIR) spectra of ND, ND-PEI and PEI were obtained by using a Perkin Elmer Spectrum 100 spectrometer equipped with an attenuated total reflection (ATR) objective.

Transmission electron microscopy (TEM) was performed on a Tecnai 20 FEG TEM with the accelerating voltage of $200 \mathrm{kV}$. A JEOL JSM7100 scanning electron microscope (SEM) was applied for the morphologies of the MMM cross-sections. Focused ion beam scanning electron microscopy (FIB-SEM) was applied to evaluate the dispersion of nanodiamond in the Pebax matrix by using an FEI SCIOS FIB/SEM dual beam system. The description of the method is shown in our previous studies [36-39]. A hole was milled on the surface of the MMM by $\mathrm{Ga}^{+}$FIB (Figure S1). Slices with a thickness of $10 \mathrm{~nm}$ were removed from the sample up to a depth of $10 \mu \mathrm{m}$ by the $\mathrm{Ga}^{+}$FIB at $30 \mathrm{kV}$ and $3 \mathrm{nA}$. Back-scattered electron (BSE) imaging mode was applied to capture the exposed cross-section images automatically. A set of cross-section SEM images was captured during this slice-and-view section with resolution of $10 \mathrm{~nm}$. The stack of these SEM images was aligned. The segmentation of different phases was identified by image thresholding. The ND fillers phase is the brightest as there is a trace of heavier elements $(\mathrm{Na}, \mathrm{Si}, \mathrm{S})$ in NDs than Pebax. The dark phase corresponds to the polymer matrix. The stack of obtained SEM images was reconstructed in three-dimensions by using the software Avizo. The volume fraction of each phase was also calculated by the software.

\subsection{Gas Permeation Test}

The gas permeation of membranes was measured in a system with variable feed pressure and constant volume, as reported elsewhere [40]. The membranes were degassed to desorb the prior permeate gas before each test fully. The tests were carried out with 2 bar feed pressure and 0.015 in the downstream at $35^{\circ} \mathrm{C}$. The diameter of the effective permeation area was $0.935 \mathrm{~cm}$. The permeability is calculated by the equation below:

$$
P=\frac{273.15 \times 10^{10}}{760 A T} \frac{V L}{\frac{P_{0} \times 76}{14.7}} \frac{d p}{d t}
$$

where $P$ is the permeability in barrer $\left(1\right.$ barrer $=1 \times 10^{-10} \mathrm{~cm}^{3}$ (STP) $\mathrm{cm} \mathrm{cm}^{-2} \mathrm{~s}^{-1} \mathrm{~cm}$ $\left.\mathrm{Hg}^{-1}\right), \mathrm{A}$ is the permeation area $\left(\mathrm{cm}^{2}\right), \mathrm{T}$ is the operation temperature $(\mathrm{K}), V$ is the dead volume of the permeate side $\left(\mathrm{cm}^{3}\right), L$ is the thickness of the membrane $(\mathrm{cm}), P_{0}$ is the feed pressure (psi), and $\mathrm{d} p / \mathrm{d} t$ is the steady rate of pressure increase in the permeate side $\left(\mathrm{mm} \mathrm{Hg} \mathrm{s}^{-1}\right)$.

The ideal selectivity for two gases is calculated by:

$$
\alpha=\frac{P_{A}}{P_{B}}
$$

where $P_{\mathrm{A}}$ and $P_{\mathrm{B}}$ are the permeability of pure gas $\mathrm{A}$ and $\mathrm{B}$, respectively. Values and error bars reported in the figures and tables are based on measurements of three different membranes.

\subsection{Felske Model}

In this work, the Felske model is used to estimate the filler-polymer interfacial properties (e.g., interface thickness and permeability) based on the the $\mathrm{CO}_{2} / \mathrm{N}_{2}$ experimental permeabilities. While detailed derivation of this model is presented in Ref. [31]. The model equations are described in the context of this work in what follows. Thus, the MMM permeability $\left(P_{m}\right)$ is defined as:

$$
P_{m}=P_{c}\left[\frac{2\left(1-\phi_{g c}\right)+\left(1+2 \phi_{g c}\right)(\eta / \gamma)}{\left(2+\phi_{g c}\right)+\left(1-\phi_{g c}\right)(\eta / \gamma)}\right]
$$


in which $\phi_{g c}=\phi_{f}+\phi_{i}$ is the volume fraction of total dispersed phase (i.e., filler particle and interfacial layer) following [41].

$$
\phi_{g c}=\frac{\phi_{f}^{N}}{\phi_{f}^{N}+\frac{\left(1-\phi_{f}^{N}\right)}{\left(1+\uparrow_{i} / r_{o}\right)^{3}}}
$$

with $\phi_{f}^{N}=\phi_{f} /\left(\phi_{f}+\phi_{c}\right)$ being the nominal filler volume fraction, $\uparrow_{i}$ the interface thickness and $r_{o}$ the particle radius. The nominal filler volume fraction $\left(\phi_{f}^{N}\right)$ in the MMM is calculated from the nominal filler weight fraction $\left(\omega_{f}\right)$ as [26]:

$$
\phi_{f}^{N}=\frac{\omega_{f}}{\omega_{f}+\frac{\rho_{f}}{\rho_{c}}\left(1-\omega_{f}\right)}
$$

where $\rho_{f}=3.5 \mathrm{~g} / \mathrm{mL}$ and $\rho_{c}=1.14 \mathrm{~g} / \mathrm{mL}$ are the nanodiamond (particle) and Pebax densities, respectively. Further, $\eta$ and $\gamma$ in Equation (3) are given by [31].

$$
\begin{gathered}
\eta=\left[2+\left(1+\uparrow_{i} / r_{o}\right)^{3}\right] \alpha_{f c}-2\left[1-\left(1+\uparrow_{i} / r_{o}\right)^{3}\right] \alpha_{i c} \\
\gamma=\left[1+2\left(1+\uparrow_{i} / r_{o}\right)^{3}\right]-\left[1-\left(1+\uparrow_{i} / r_{o}\right)^{3}\right] \alpha_{f i}
\end{gathered}
$$

where $\alpha_{f c}=P_{f} / P_{c}, \alpha_{i c}=P_{i} / P_{c}$ and $\alpha_{f i}=P_{f} / P_{i}$. In Equations (3)-(7), $P$ and $\phi$ denote the permeability and volume fraction, respectively, and superscripts/subscripts $f, c, i, m$ and $g$ denote filler phase, continuous phase, interface, MMM, and combined filler phase and interface composite, respectively.

\section{Results and Discussion}

\subsection{Preparation and Characterisation of Modified Nanodiamonds}

Figure 1 shows the TEM images of ND and ND-PEI. The ND expresses a spherical shape with a size of 5-10 $\mathrm{nm}$. After the PEI impregnation, the size and shape of the ND particles did not change significantly. The loading of PEI in ND-PEI was calculated by TGA. Two main degradation stages were observed in the TGA result (Figure S2). The first stage of degradation from $150{ }^{\circ} \mathrm{C}$ to $350^{\circ} \mathrm{C}$ should correspond to the degradation of PEI on the ND. The loading of PEI in ND-PEI is around 10\%. The content of carbon, hydrogen and nitrogen in ND and ND-PEI samples has been conducted by an elemental analyser to confirm the success of the surface modification of ND by PEI and the results are shown in Table 1. Compared to the ND, the ND-PEI particles show a higher ratio of nitrogen and hydrogen elements, as would be expected under successful coating. The introduction of PEI layer onto the ND surface increased the amount of $\mathrm{N}$ and $\mathrm{H}$ elements. These results suggest that the PEI has been successfully incorporated on the ND surface.

X-ray photoelectron spectra (XPS) also has been applied to confirm the PEI layer coated on the ND. Figure 2a shows the survey scans of the ND and ND-PEI samples. ND-PEI shows higher (4.86 at \%) nitrogen ratio than ND (1.83 at \%), which is consistent with the elemental analysis results. For the N1s spectra (Figure $2 b$ ), the ND sample exhibited two peaks at around 397.8 and $402.0 \mathrm{eV}$, which correspond to the heteroatom nitrogen on the ND surface structure [42,43]. For the ND-PEI sample, four peaks were found at 397.8, 398.6, 399.7, and $402.0 \mathrm{eV}$. The two peaks found in ND spectrum at 397.8 and $402.0 \mathrm{eV}$ also appeared in the spectrum of ND-PEI. The two new peaks at 398.6 and $399.7 \mathrm{eV}$ correspond to amine and protonated amine groups in PEI chains, respectively. These XPS results demonstrate NDs are covered by the PEI. Fourier-transform infrared (FTIR) spectra of ND, NE-PEI and PEI were investigated (Figure S3). Compared with the spectrum of ND, the decrease of the peak at $1629 \mathrm{~cm}^{-1}$ (O-H bending, hydroxyl) and the increase of the peak at $1662 \mathrm{~cm}^{-1}$ (C=O stretching of amide) [44] were observed in the spectrum of ND-PEI, 
revealing the presence of PEI and its interaction with the oxidised surface of ND through the surface $-\mathrm{OH}$ groups.
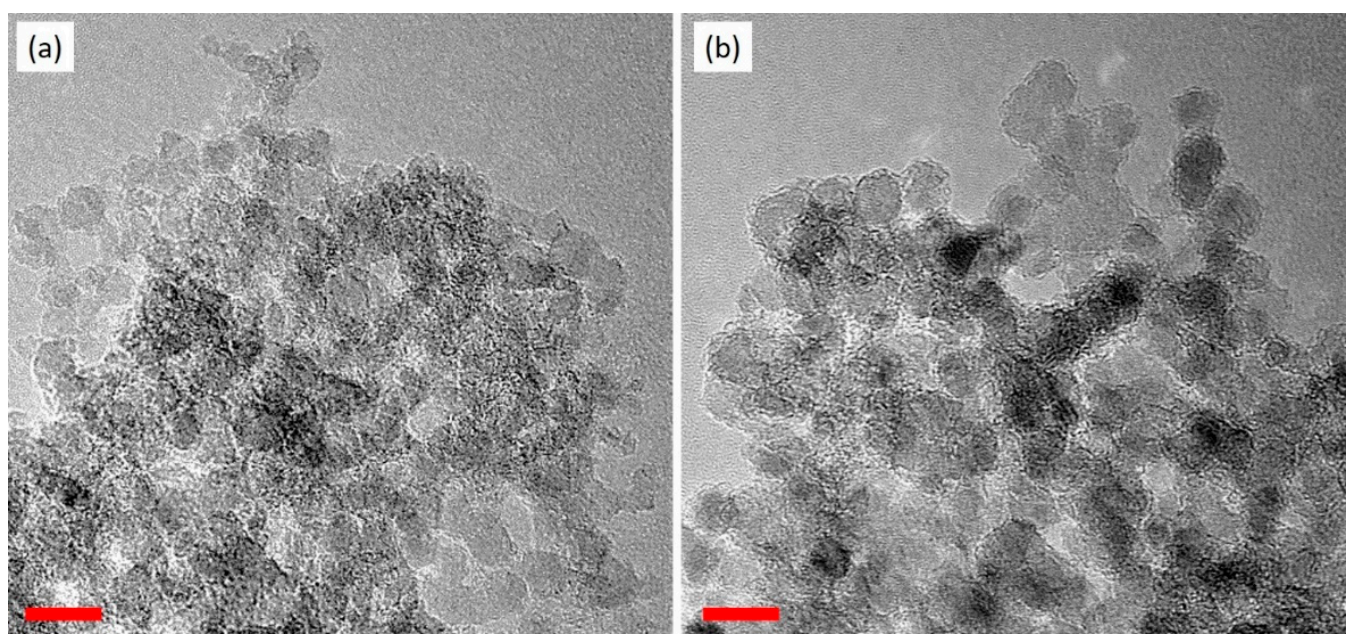

Figure 1. TEM images of (a) ND and (b) ND-PEI. The red lines are the scale bars. Both scale bars are $10 \mathrm{~nm}$.

Table 1. Elemental weight ratio of pristine ND and ND-PEI particles.

\begin{tabular}{cccc}
\hline \multirow{2}{*}{ Samples } & \multicolumn{3}{c}{ Elemental Ratio (wt.\%) } \\
\cline { 2 - 4 } & $\mathbf{N}$ & $\mathbf{C}$ & $\mathbf{H}$ \\
\hline ND & 1.61 & 86.27 & 0.64 \\
ND-PEI & 4.34 & 83.94 & 1.25 \\
\hline
\end{tabular}
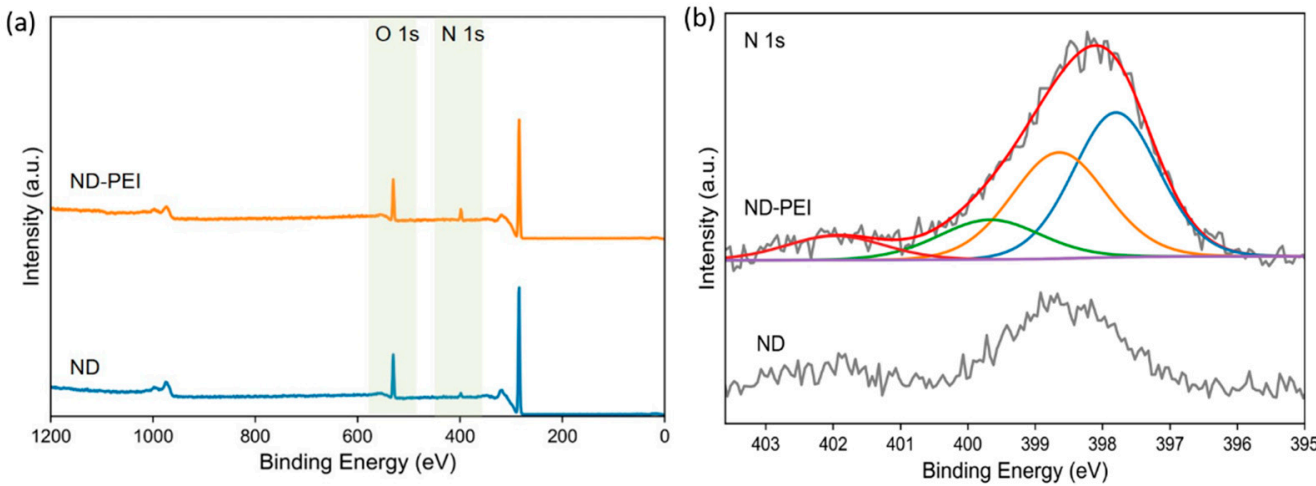

Figure 2. XPS spectra of ND and ND-PEI. (a) Survey scans the ranging from 0 to $1200 \mathrm{eV}$. (b) N1s.

\subsection{Characterisation of $M M M s$}

The transport performance and the integrity of the membrane rely on the filler dispersion and the filler/matrix adhesion. The interfacial morphology of Pebax/ND MMMs and Pebax/ND-PEI MMMs were investigated by FESEM and the images are displayed in Figures 3 and 4. Compared to the pristine Pebax membrane (Figure S4a,b), poor dispersion of ND in the Pebax matrix was clearly observed in the Pebax/ND MMMs (Figure 3), as indicated by large aggregates of ND (identified by arrows) in the matrix. It seems that the oxygen-containing functional groups on the ND surface do not provide appreciable assistance in dispersing the ND in the Pebax matrix. Without further surface modification, the ND tends to form agglomerations easily. The aggregation of ND can cause the formation of non-selective interfacial voids, leading to the deterioration in gas selectivity of the membrane. With further increases in the ND loading, unsurprisingly, more aggregate clusters with larger sizes were observed (Figure S4c,d). 

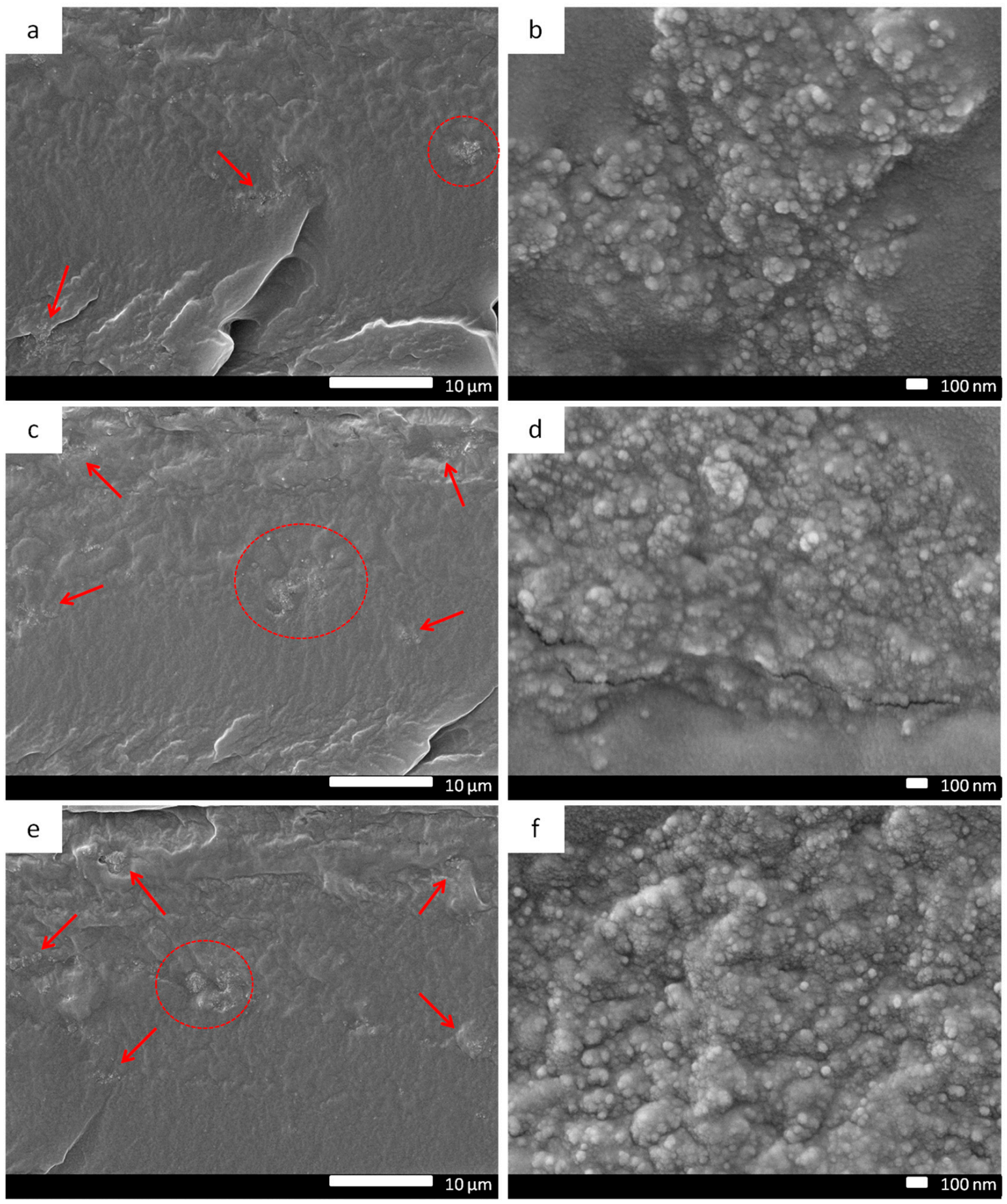

Figure 3. Membrane cross-section SEM images of Pebax/ND MMMs with different ND ratio: 0.1 wt.\% (a,b), 0.5 wt.\% (c,d), 1.0 wt.\% (e,f), arrows point to ND aggregate clusters in the MMMs.

In the case of Pebax/ND-PEI MMMs (Figure 4 and Figure S4e,f), the individual ND-PEI particles were less visible in the matrix. The roughness of the Pebax/ND-PEI MMMs was significantly increased compared to the neat Pebax membranes. In an obvious contrast to the Pebax/ND MMMs (Figure 3), there was no visible ND agglomeration at the cross-section of the Pebax/ND-PEI MMMs even with the highest loading of ND-PEI (1.5 wt.\%) (Figure S4e,f). These SEM images indicate an improved dispersion of ND in polymer matrix was achieved. The improvement in dispersion of ND-PEI with the polymer matrix is attributed to the presence of the PEI layer on ND surface. The PEI layer acts as 
a polymeric compatibiliser, which can improve the compatibility between the crystalline carbon structure of ND and the rubbery polymeric nature of Pebax, preventing the filler aggregation and leading to an increase in dispersibility of ND in the Pebax matrix.
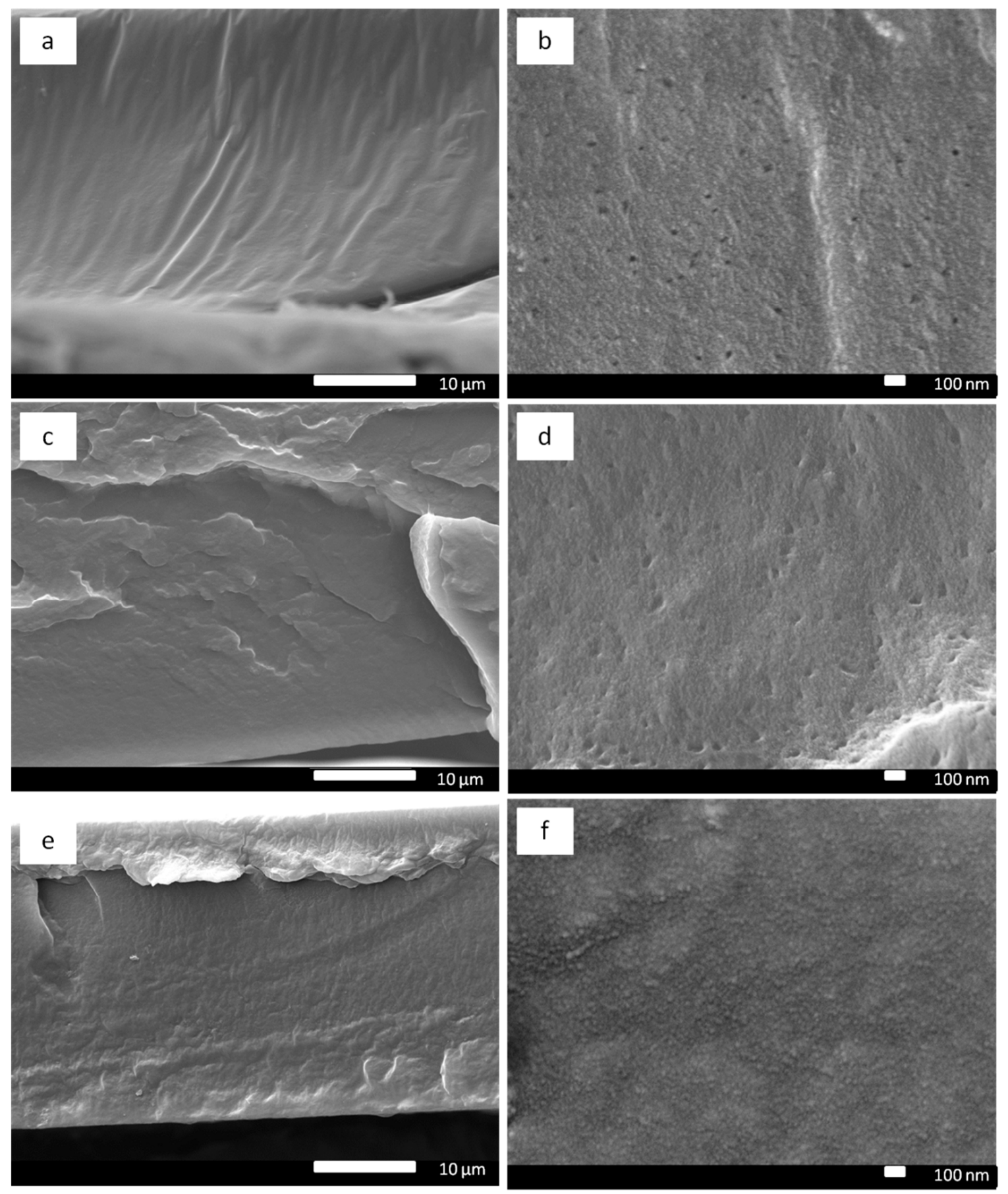

Figure 4. Membrane cross-section SEM images of Pebax/ND-PEI MMMs with different ND ratio: 0.1 wt. \% (a,b), 0.5 wt. \% (c,d), $1.0 \mathrm{wt} . \%(\mathbf{e}, \mathbf{f})$.

To further achieve more comprehensive structural information of the ND MMMs, the distribution of ND and ND-PEI particles within the Pebax matrix was investigated by focused ion beam SEM (FIB-SEM). Figure 5a,b shows the 3D reconstructed images of the filler distribution in Pebax/ND and Pebax/ND-PEI MMMs. As also observed in the SEM images above (Figure 3), the ND particles formed large agglomerates in the MMMs, while 
in contrast, ND-PEI aggregates were far less likely to be observed. Due to the presence of PEI, ND-PEI exhibit better dispersion than unmodified ND, presented as much smaller particles and aggregates. Besides, as the resolution of FIB observation is $10 \mathrm{~nm}, \mathrm{ND}$ and ND-PEI particles with the size of $10 \mathrm{~nm}$ or below are hardly detected, which leads to fewer ND particles observed in the FIB images. Based on the 3D image analysis, the ND agglomerates size distributions in the MMMs were calculated and reported in Figure 5c. For the Pebax/ND MMMs, a majority of the ND was in the form of large-volume particles $\left(10^{4}-10^{6} \mathrm{~nm}^{3}\right)$, while the small volume particles only occupy a minor fraction, which indicated the agglomeration of ND particles in the MMMs. On the other hand, the ND-PEI exhibited a more significant number of smaller particles (as measured by volume), indicating less agglomeration and better dispersion of ND-PEI in the polymer matrix due to the incorporation of PEI.
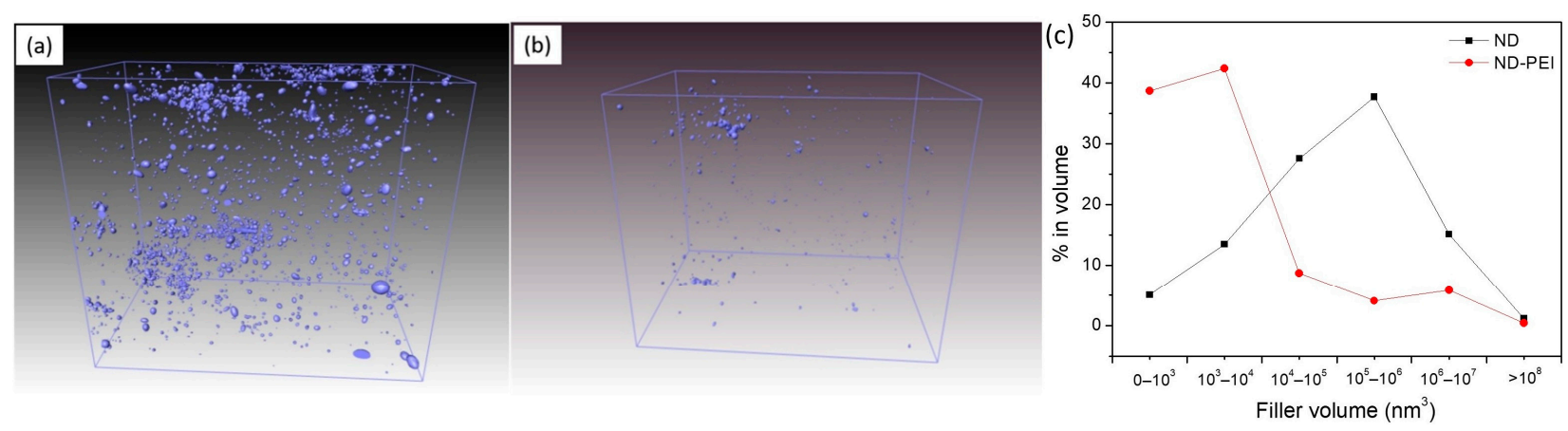

Figure 5. The FIB surface rendered view of (a) Pebax/ND MMM, (b) Pebax/ND-PEI MMM, and (c) filler particle size distributions as derived from image analysis of the FIB-SEM tomogram.

\subsection{Gas Separation Performance}

The ideal gas separation performance of the pristine Pebax membranes, Pebax/ND MMMs and Pebax/ND-PEI MMMs were investigated by single gas permeability measurements and the results are shown in Figure 6 and Table S1. The pristine Pebax membrane showed a $\mathrm{CO}_{2}$ permeability of 56 barrer with a $\mathrm{CO}_{2} / \mathrm{N}_{2}$ selectivity of 40.60 , which is consistent with a previous study [45]. At a lower loading of ND $(0.1 \mathrm{wt} . \%, 0.5 \mathrm{wt} . \%$, $1 \mathrm{wt} . \%$ ), the $\mathrm{CO}_{2}$ and $\mathrm{N}_{2}$ permeability of ND MMMs decreased after the introduction of $\mathrm{ND}$ when compared to the neat Pebax membranes, with the $\mathrm{CO}_{2} / \mathrm{N}_{2}$ selectivity reduced simultaneously. The presence of ND in the polymer matrix may occupy free volume between the polymer chains, which combined with the non-porous nature of ND, prevented gas diffusion through the membrane, leading to a reduction in gas permeability. Further, non-selective voids may form in the ND aggregates due to poor filler dispersion, resulting in the deterioration of $\mathrm{CO}_{2} / \mathrm{N}_{2}$ selectivity of the MMMs. At $1.5 \mathrm{wt} . \%$ loading of $\mathrm{ND}$, the Pebax/ND MMMs exhibited a higher $\mathrm{CO}_{2}$ permeability as well as better $\mathrm{CO}_{2} / \mathrm{N}_{2}$ selectivity compared to the lower ND loading MMMs. At high loading, ND particles tend to form big clusters of agglomeration, which disrupted the polymer structure and created the non-selective voids inside the MMMs that in turn increase the permeability of both $\mathrm{CO}_{2}$ and $\mathrm{N}_{2}$ through the membranes.

In the case of MMMs with ND-PEI (Figure 6), at $0.1 \mathrm{wt} . \%$ loading of ND-PEI, the gas permeability of MMMs decreased while the $\mathrm{CO}_{2} / \mathrm{N}_{2}$ selectivity slightly reduced. In this case, the incorporation of PEI may improve the interfacial adhesion of ND and the Pebax matrix, which results in the reduction of free volume in the MMMs due to the occupation of ND-PEI particles. This result leads to the deterioration of both $\mathrm{CO}_{2}$ and $\mathrm{N}_{2}$ permeability through the MMMs. At lower loading of ND-PEI, the amount of PEI incorporated in the MMMs may not be enough to enhance the $\mathrm{CO}_{2}$ permeability to overcome the deterioration above, leading to a slight reduction in the case of MMMs with ND-PEI action in $\mathrm{CO}_{2} / \mathrm{N}_{2}$ selectivity. When the loading of ND-PEI is increased (up to $1 \mathrm{wt} . \%$ ), both $\mathrm{CO}_{2}$ permeability 
and $\mathrm{CO}_{2} / \mathrm{N}_{2}$ selectivity of the Pebax/ND-PEI MMMs were improved compared to the pristine Pebax. The introduction of the PEI layer significantly enhanced the interfacial interaction between ND particles and polymer matrix, which prevents the formation of aggregates and reduces the formation of non-selective voids, leading to better $\mathrm{CO}_{2} / \mathrm{N}_{2}$ separation performance. Furthermore, the PEI layer on the ND surface can also act as the " $\mathrm{CO}_{2}$ carrier agent" due to the amine functional groups, which further enhances the solubility of $\mathrm{CO}_{2}$ through the MMMs and subsequently improves the $\mathrm{CO}_{2} / \mathrm{N}_{2}$ selectivity. At 1.5 wt.\% loading of ND-PEI, the MMMs show higher permeability for both $\mathrm{CO}_{2}$ and $\mathrm{N}_{2}$ while the $\mathrm{CO}_{2} / \mathrm{N}_{2}$ decreased (37.09), which can be due to the slight formation of the ND aggregations caused by the higher concentration of ND in the polymer matrix. This is in agreement with the SEM investigation. We conclude that depositing PEI on ND presents a modification method to solve the nano-filler aggregation problem and improve the filler/polymer interface, leading to a significant improvement in membrane performance.
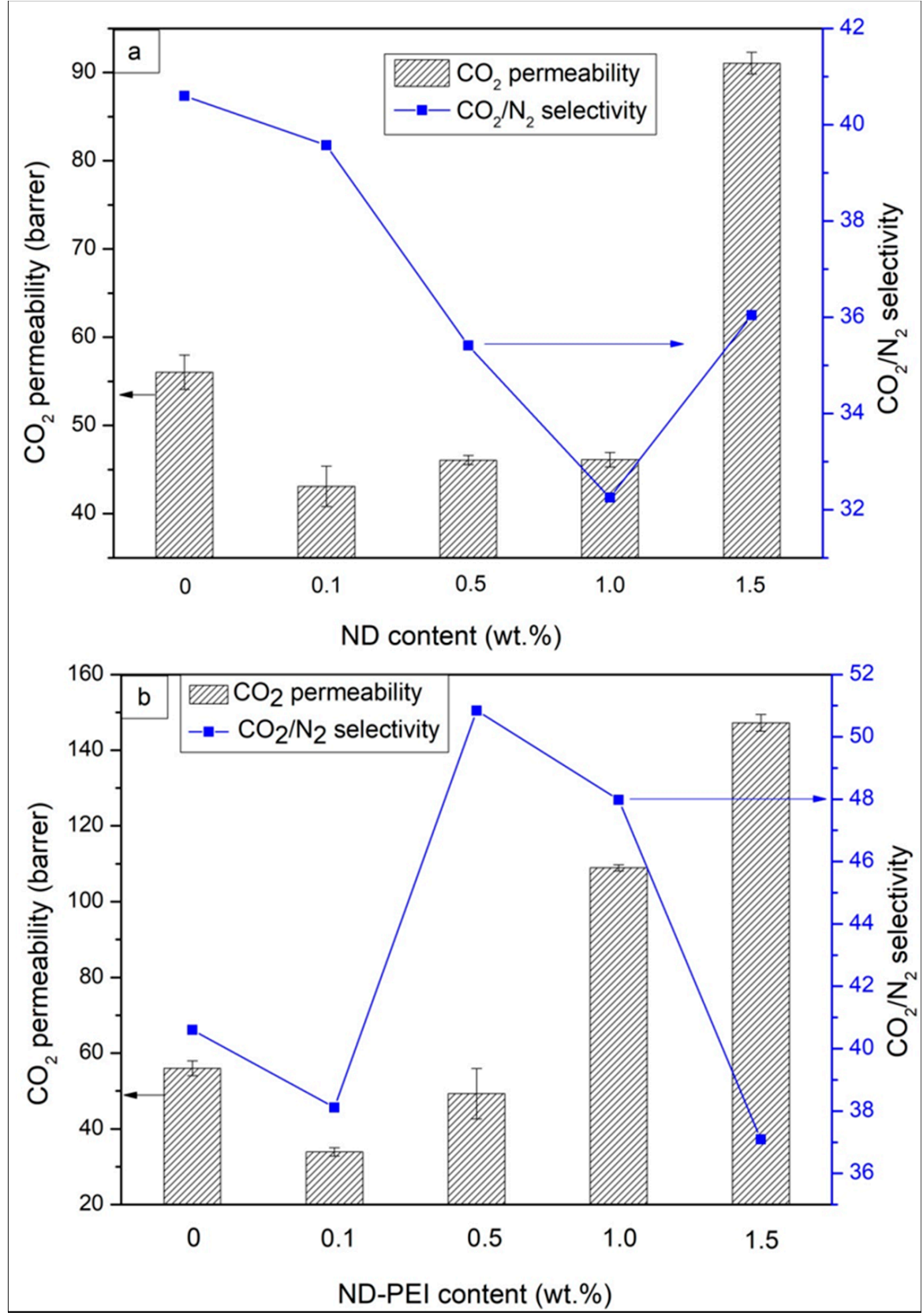

Figure 6. Gas separation performance of Pebax/ND MMMs (a) and Pebax/ND-PEI MMMs (b). 


\subsection{Modelling}

Finally, because Figures 5 and 6 suggest that surface modification of ND with PEI improves the filler/polymer interfacial properties, leading to a significant improvement in the MMM performance, the interfacial permeabilities $\left(P_{i}\right)$ of each gas are calculated using the Felske model [31] summarized in Section 2.6. This model has been shown to provide a good approximation of interfacial properties (i.e., interface thickness and permeability) when particle loading is low (less than 10-15\%) in the MMM, as discussed elsewhere [32,33]. Figure 7 depicts a comparison of the experimental permeabilities (open star symbols) to those based on the Felske model (closed circle symbols) with an increase of ND loading in the MMM. Figure 7a,b depicts $\mathrm{CO}_{2}$ and $\mathrm{N}_{2}$ permeabilities in the Pebax/ND MMMs while Figure 7c,d depicts those in Pebax/ND-PEI MMMs, respectively. It is noted that while abscissas in Figure 7 correspond to the nominal ND percentage weight fraction (c.f. Section 2.3), pristine Pebax and ND particle densities are used to calculate the ND volume fraction in the MMMs and thus solve the Felske model for the interfacial permeability $\left(P_{i}\right)$ in each case (c.f. Section 2.6). Further, each theoretical data point is accompanied by the calculated $P_{i}$ considering the ND permeability equal $P_{f}=0$ (nonporous filler) and a ratio of the interface thickness to ND size (radius) of $\uparrow_{i} / r_{o}=40$ in the Felske model. For this membrane system, the interfacial permeabilities are found to be insensitive to the variation of this ratio, as shown in the Supplementary Material (Figure S5) for $\uparrow_{i} / r_{o}$ between 20-1000, with no suitable model solution found for ratios below 20. This tendency suggests that the ratio of the interface thickness to the particle ratio is likely to be greater than 20 for this system. While this result is in agreement with recently reported ND structures [46,47], having ND core sizes relatively smaller than their shells, detailed characterisation of the Pebax/ND-PEI interfacial morphology will be required to estimate the interface thickness quantitively. In Figure $7 \mathrm{a}, \mathrm{b}$ the interfacial permeabilities are slightly lower than those in neat Pebax at low loadings (0.1 wt.\%, $0.5 \mathrm{wt} . \%$, and $1.0 \mathrm{wt} . \%)$. A similar tendency is depicted in Figure 7c,d at low loadings ( $0.1 \mathrm{wt} . \%$ and $0.5 \mathrm{wt} . \%)$ for both gases in Pebax/ND-PEI MMMs, which suggests the absence of interfacial voids for these membranes. Alternatively, the $\mathrm{CO}_{2}$ and $\mathrm{N}_{2}$ interfacial permeabilities in Figure $7 \mathrm{a}, \mathrm{b}$ for the highest ND loading $(1.5 \mathrm{wt} . \%)$ are about twice those in the neat Pebax membranes, which corroborates the presence of non-selective interfacial voids in the MMM due to particle agglomeration. This tendency has also been recently recognised in the removal of lead ions from aqueous solutions in nanodiamond/chitosan-polyvinyl alcohol (ND/CSPVA) membranes, in which particle agglomeration was observed for ND loadings of 1.5 wt.\% [48]. Further, while the $\mathrm{CO}_{2}$ permeability in Figure $7 \mathrm{c}$ is also about twice that in the neat polymer when the ND loading is $1.0 \%, \mathrm{~N}_{2}$ in Figure $7 \mathrm{~d}$ is about the same as in the neat Pebax membrane. This tendency corroborates that the increase of the $\mathrm{CO}_{2} / \mathrm{N}_{2}$ selectivity, when the ND surface is modified with PEI, is associated with an increase in the solubility of $\mathrm{CO}_{2}$ due to the presence of PEI amine functional groups, which improve the affinity to $\mathrm{CO}_{2}$ without enhancing the $\mathrm{N}_{2}$ adsorption capacity. Alternatively, when ND loading is $1.5 \mathrm{wt} . \%$ in Figure $7 \mathrm{c}$, d both $\mathrm{CO}_{2}$ and $\mathrm{N}_{2}$ permeabilities are significantly larger than in neat Pebax, which is associated with a combination of effects in the membrane system. On one hand, the $\mathrm{CO}_{2}$ has a better affinity for the PEI amine functional groups, which leads to an increase of the interfacial permeability. On the other, this MMM exhibited particle agglomeration, leading to the formation of non-selective interfacial voids and thus a further increase of both $\mathrm{CO}_{2}$ and $\mathrm{N}_{2}$ interfacial permeabilities. 

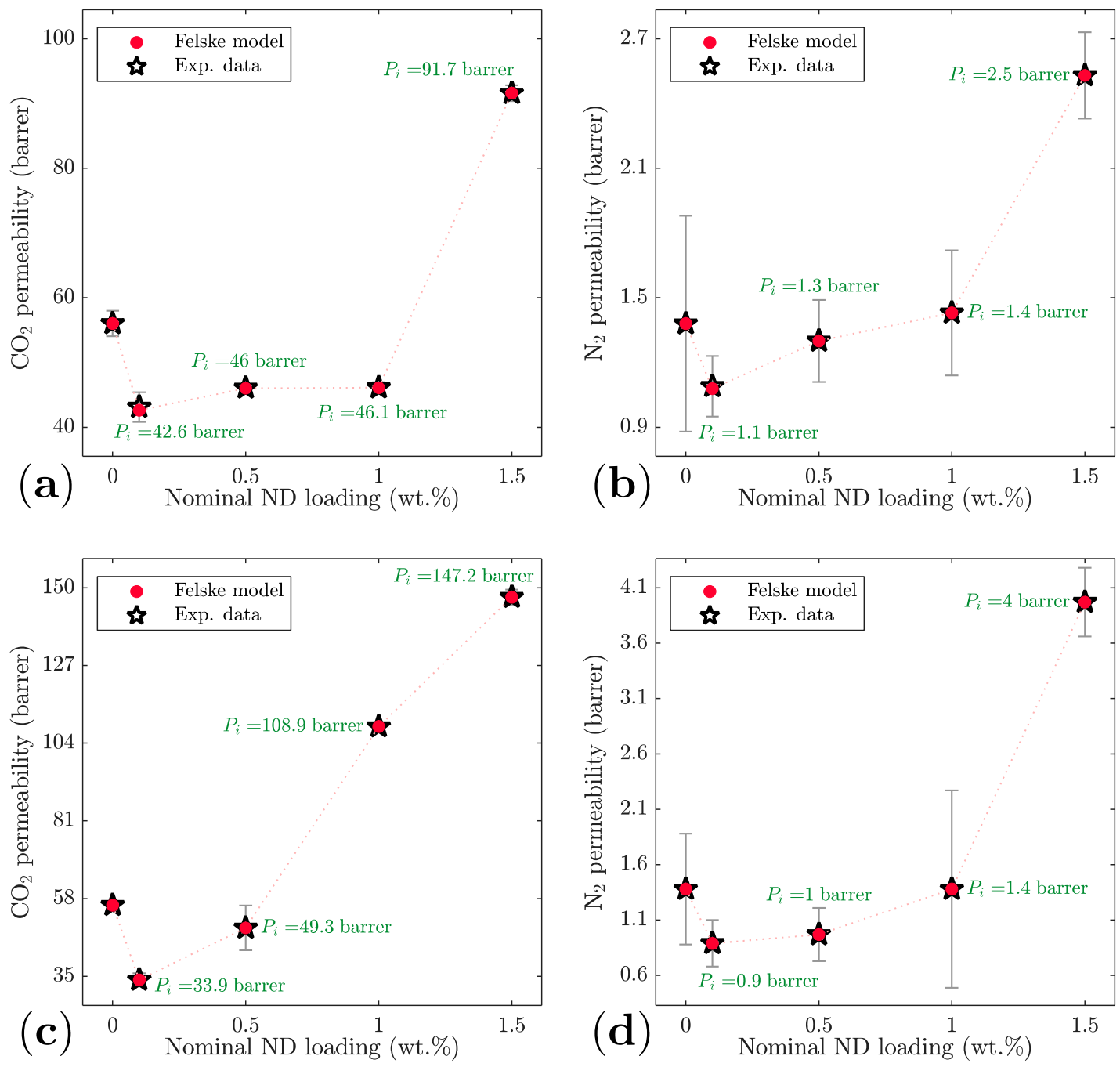

Figure 7. Predicted $\mathrm{CO}_{2}$ and $\mathrm{N}_{2} \mathrm{MMM}$ and interfacial permeabilities with increase of $\mathrm{ND}$ loading. (a) $\mathrm{CO}_{2}$ in $\mathrm{Pebax} / \mathrm{ND}$, (b) $\mathrm{N}_{2}$ in Pebax/ND, (c) $\mathrm{CO}_{2}$ in Pebax/ND-PEI (d) $\mathrm{N}_{2}$ in Pebax/ND-PEI. Dotted lines correspond to a guide for the eye. Error bars correspond to experimental error bars.

\section{Conclusions}

In this study, mixed matrix membranes based on ND nanoparticles and Pebax matrix for $\mathrm{CO}_{2} / \mathrm{N}_{2}$ separation were obtained. Surface modification of ND by PEI decoration has been conducted, of which success was confirmed by elemental analysis, XPS and FTIR. The presence of PEI layer on ND surface effectively improved the interfacial adhesion and dispersion of ND in the Pebax matrix, which were clearly indicated by SEM observation. The ideal selectivity of $\mathrm{CO}_{2} / \mathrm{N}_{2}$ was also significantly improved with the incorporation of PEI up to 1 wt.\% of ND-PEI filler in the MMMs, due to the "CO $\mathrm{CO}_{2}$ carrier" role of PEI, which was corroborated through the calculation of the $\mathrm{CO}_{2}$ and $\mathrm{N}_{2}$ interfacial permeabilities using the Felske model. These results expressed an effective modification approach for applying the potential non-porous ND into gas separation membrane application. Moreover, the PEI decoration method can be comparable with a wide range of nanofillers for improved distribution in a polymer matrix.

Supplementary Materials: The following are available online at https:/ /www.mdpi.com/article/10 $.3390 /$ membranes11050328/s1, Figure S1. Typical FIB-SEM images of Pebax/ND 1.5 wt.\% MMM: (a) FIB milling trend and (b) cross-sectional image in BSE mode, Figure S2. Thermogravimetric analysis of ND-PEI at a ramp rate of $20{ }^{\circ} \mathrm{C}$ min-1, Figure S3. Fourier-transform infrared spectra 
of ND, ND-PEI and PEI., Figure S4. Membrane cross-section SEM images of pristine Pebax $(a, b)$, Pebax/ND 1.5 wt.\% (c, d) and Pebax/ND-PEI 1.5 wt.\% (e, f) MMMs. Figure S5. Predicted CO2 and $\mathrm{N} 2$ interfacial and MMM permeabilities with increase the ratio of the interface thickness to particle size. (a) CO2 interfacial permeability (b) CO2 permeability in Pebax/ND-PEI and, (c) N2 interfacial permeability (d) N2 permeability in Pebax/ND-PEI., Table S1. Gas permeability and selectivity of pure Pebax membrane, Pebax/ND MMMs and Pebax/ND-PEI MMMs.

Author Contributions: Conceptualization, R.L. and S.S.; methodology, M.-T.V., R.L. and S.S.; validation, R.L., G.M.M.-B., M.L. and S.S.; formal analysis, M.-T.V., G.M.M.-B., R.L. and M.L.; investigation, M.-T.V., G.M.M.-B. and R.L.; writing-original draft preparation, M.-T.V. and R.L.; writing-review and editing, S.S. and S.K.B.; visualization, M.-T.V.; supervision, R.L., S.K.B. and S.S.; project administration, S.S. and S.K.B.; funding acquisition, S.K.B. and S.S. All authors have read and agreed to the published version of the manuscript.

Funding: The authors acknowledge the financial support from the Australian Research Council Discovery Project (150101996).

Institutional Review Board Statement: Not applicable.

Informed Consent Statement: Not applicable.

Acknowledgments: The authors acknowledge the Centre for Microscopy and Microanalysis (CMM) at The University of Queensland for providing facilities and support in characterisations in this work. M.T. Vu also acknowledges The University of Queensland postgraduate scholarship.

Conflicts of Interest: The authors declare no competing financial interest.

\section{References}

1. Robeson, L.M. The Upper Bound Revisited. J. Membr. Sci. 2008, 320, 390-400. [CrossRef]

2. Park, H.B.; Kamcev, J.; Robeson, L.M.; Elimelech, M.; Freeman, B.D. Maximizing the right stuff: The trade-off between membrane permeability and selectivity. Science 2017, 356, eaab0530. [CrossRef]

3. Chung, T.-S.; Jiang, L.Y.; Li, Y.; Kulprathipanja, S. Mixed matrix membranes (MMMs) comprising organic polymers with dispersed inorganic fillers for gas separation. Prog. Polym. Sci. 2007, 32, 483-507. [CrossRef]

4. Dong, G.; Li, H.; Chen, V. Challenges and opportunities for mixed-matrix membranes for gas separation. J. Mater. Chem. A 2013, 1, 4610-4630. [CrossRef]

5. Lin, R.; Ge, L.; Diao, H.; Rudolph, V.; Zhu, Z. Ionic Liquids as the MOFs/Polymer Interfacial Binder for Efficient Membrane Separation. ACS Appl. Mater. Interfaces 2016, 8, 32041-32049. [CrossRef]

6. Vinoba, M.; Bhagiyalakshmi, M.; Alqaheem, Y.; Alomair, A.A.; Pérez, A.; Rana, M.S. Recent progress of fillers in mixed matrix membranes for $\mathrm{CO}_{2}$ separation: A review. Sep. Purif. Technol. 2017, 188, 431-450. [CrossRef]

7. Lin, R.; Hernandez, B.; Ge, L.; Zhu, Z. Metal organic framework based mixed matrix membranes: An overview on filler/polymer interfaces. J. Mater. Chem. A 2018, 6, 293-312. [CrossRef]

8. Krueger, A. Diamond Nanoparticles: Jewels for Chemistry and Physics. Adv. Mater. 2008, 20, 2445-2449. [CrossRef]

9. Behler, K.D.; Stravato, A.; Mochalin, V.; Korneva, G.; Yushin, G.; Gogotsi, Y. Nanodiamond-Polymer Composite Fibers and Coatings. ACS Nano 2009, 3, 363-369. [CrossRef]

10. Branson, B.T.; Beauchamp, P.S.; Beam, J.C.; Lukehart, C.M.; Davidson, J.L. Nanodiamond Nanofluids for Enhanced Thermal Conductivity. ACS Nano 2013, 7, 3183-3189. [CrossRef]

11. Liu, W.; Naydenov, B.; Chakrabortty, S.; Wuensch, B.; Hübner, K.; Ritz, S.; Cölfen, H.; Barth, H.; Koynov, K.; Qi, H.; et al. Fluorescent Nanodiamond-Gold Hybrid Particles for Multimodal Optical and Electron Microscopy Cellular Imaging. Nano Lett. 2016, 16, 6236-6244. [CrossRef] [PubMed]

12. Zhang, T.; Liu, G.-Q.; Leong, W.-H.; Liu, C.-F.; Kwok, M.-H.; Ngai, T.; Liu, R.-B.; Li, Q. Hybrid nanodiamond quantum sensors enabled by volume phase transitions of hydrogels. Nat. Commun. 2018, 9, 3188. [CrossRef] [PubMed]

13. Zhang, X.-Q.; Chen, M.; Lam, R.; Xu, X.; Osawa, E.; Ho, D. Polymer-Functionalized Nanodiamond Platforms as Vehicles for Gene Delivery. ACS Nano 2009, 3, 2609-2616. [CrossRef] [PubMed]

14. Zhang, X.-Q.; Lam, R.; Xu, X.; Chow, E.K.; Kim, H.-J.; Ho, D. Multimodal Nanodiamond Drug Delivery Carriers for Selective Targeting, Imaging, and Enhanced Chemotherapeutic Efficacy. Adv. Mater. 2011, 23, 4770-4775. [CrossRef]

15. Faklaris, O.; Joshi, V.; Irinopoulou, T.; Tauc, P.; Sennour, M.; Girard, H.; Gesset, C.; Arnault, J.-C.; Thorel, A.; Boudou, J.-P.; et al. Photoluminescent Diamond Nanoparticles for Cell Labeling: Study of the Uptake Mechanism in Mammalian Cells. ACS Nano 2009, 3, 3955-3962. [CrossRef]

16. Girard, H.A.; Arnault, J.C.; Perruchas, S.; Saada, S.; Gacoin, T.; Boilot, J.P.; Bergonzo, P. Hydrogenation of nanodiamonds using MPCVD: A new route toward organic functionalization. Diam. Relat. Mater. 2010, 19, 1117-1123. [CrossRef]

17. Mochalin, V.N.; Shenderova, O.; Ho, D.; Gogotsi, Y. The properties and applications of nanodiamonds. Nat. Nanotechnol. 2012, 7, 11-23. [CrossRef] 
18. Kumar, S.; Nehra, M.; Kedia, D.; Dilbaghi, N.; Tankeshwar, K.; Kim, K.-H. Nanodiamonds: Emerging face of future nanotechnology. Carbon 2019, 143, 678-699. [CrossRef]

19. Kausar, A. Nanodiamond integrating poly(methyl methacrylate) nanocomposites intending for technological innovations. Mater. Res. Innov. 2020, 1-10. [CrossRef]

20. Zhang, Y.; Choi, J.R.; Park, S.-J. Thermal conductivity and thermo-physical properties of nanodiamond-attached exfoliated hexagonal boron nitride/epoxy nanocomposites for microelectronics. Compos. Part A Appl. Sci. Manuf. 2017, 101, 227-236. [CrossRef]

21. Etemadi, H.; Yegani, R.; Seyfollahi, M. The effect of amino functionalized and polyethylene glycol grafted nanodiamond on anti-biofouling properties of cellulose acetate membrane in membrane bioreactor systems. Sep. Purif. Technol. 2017, 177, 350-362. [CrossRef]

22. Li, Y.; Huang, S.; Zhou, S.; Fane, A.G.; Zhang, Y.; Zhao, S. Enhancing water permeability and fouling resistance of polyvinylidene fluoride membranes with carboxylated nanodiamonds. J. Membr. Sci. 2018, 556, 154-163. [CrossRef]

23. Bhadra, M.; Roy, S.; Mitra, S. Nanodiamond immobilized membranes for enhanced desalination via membrane distillation. Desalination 2014, 341, 115-119. [CrossRef]

24. Qin, D.; Huang, G.; Terada, D.; Jiang, H.; Ito, M.M.; Gibbons, A.H.; Igarashi, R.; Yamaguchi, D.; Shirakawa, M.; Sivaniah, E.; et al. Nanodiamond mediated interfacial polymerization for high performance nanofiltration membrane. J. Membr. Sci. 2020, 603, 118003. [CrossRef]

25. Polotskaya, G.A.; Avagimova, N.V.; Toikka, A.M.; Tsvetkov, N.V.; Lezov, A.A.; Strelina, I.A.; Gofman, I.V.; Pientka, Z. Optical, mechanical, and transport studies of nanodiamonds/poly(phenylene oxide) composites. Polym. Compos. 2018, 39, 3952-3961. [CrossRef]

26. Avagimova, N.; Polotskaya, G.; Toikka, A.; Pulyalina, A.; Morávková, Z.; Trchová, M.; Pientka, Z. Effect of nanodiamond additives on the structure and gas-transport properties of a poly(phenylene-isophtalamide) matrix. J. Appl. Polym. Sci. 2018, 135, 46320. [CrossRef]

27. Aroon, M.A.; Ismail, A.F.; Matsuura, T.; Montazer-Rahmati, M.M. Performance Studies of Mixed Matrix Membranes for Gas Separation: A review. Sep. Purif. Technol. 2010, 75, 229-242. [CrossRef]

28. Molavi, H.; Shojaei, A.; Mousavi, S.A. Improving mixed-matrix membrane performance via PMMA grafting from functionalized NH2-UiO-66. J. Mater. Chem. A 2018, 6, 2775-2791. [CrossRef]

29. Sutrisna, P.D.; Hou, J.; Li, H.; Zhang, Y.; Chen, V. Improved operational stability of Pebax-based gas separation membranes with ZIF-8: A comparative study of flat sheet and composite hollow fibre membranes. J. Membr. Sci. 2017, 524, 266-279. [CrossRef]

30. Feng, S.; Ren, J.; Li, Z.; Li, H.; Hua, K.; Li, X.; Deng, M. Poly(amide-12-b-ethylene oxide)/glycerol triacetate blend membranes for $\mathrm{CO}_{2}$ separation. Int. J. Greenh. Gas Control 2013, 19, 41-48. [CrossRef]

31. Felske, J. Effective thermal conductivity of composite spheres in a continuous medium with contact resistance. Int. J. Heat Mass Transf. 2004, 47, 3453-3461. [CrossRef]

32. Vinh-Thang, H.; Kaliaguine, S. Predictive Models for Mixed-Matrix Membrane Performance: A Review. Chem. Rev. 2013, 113, 4980-5028. [CrossRef]

33. Monsalve-Bravo, G.M.; Bhatia, S.K. Modeling Permeation through Mixed-Matrix Membranes: A Review. Processes 2018, 6, 172. [CrossRef]

34. Monsalve-Bravo, G.M.; Dutta, R.C.; Bhatia, S.K. Multiscale simulation of gas transport in mixed-matrix membranes with interfacial polymer rigidification. Microporous Mesoporous Mater. 2020, 296, 109982. [CrossRef]

35. Inel, G.A.; Ungureau, E.-M.; Varley, T.S.; Hirani, M.; Holt, K.B. Solvent-surface interactions between nanodiamond and ethanol studied with in situ infrared spectroscopy. Diam. Relat. Mater. 2016, 61, 7-13. [CrossRef]

36. Lin, R.; Ge, L.; Diao, H.; Rudolph, V.; Zhu, Z. Propylene/propane selective mixed matrix membranes with grape-branched MOF/CNT filler. J. Mater. Chem. A 2016, 4, 6084-6090. [CrossRef]

37. Ge, L.; Lin, R.; Wang, L.; Rufford, T.E.; Villacorta, B.; Liu, S.; Liu, L.X.; Zhu, Z. Surface-etched halloysite nanotubes in mixed matrix membranes for efficient gas separation. Sep. Purif. Technol. 2017, 173, 63-71. [CrossRef]

38. Vu, M.-T.; Lin, R.; Diao, H.; Zhu, Z.; Bhatia, S.K.; Smart, S. Effect of ionic liquids (ILs) on MOFs/polymer interfacial enhancement in mixed matrix membranes. J. Membr. Sci. 2019, 587, 117157. [CrossRef]

39. Lin, R.; Hou, J.; Li, M.; Wang, Z.; Ge, L.; Li, S.; Smart, S.; Zhu, Z.; Bennett, T.D.; Chen, V. Interfacial engineering of a polymer-MOF composite by in situ vitrification. Chem. Commun. 2020, 56, 3609-3612. [CrossRef]

40. Ge, L.; Zhu, Z.; Li, F.; Liu, S.; Wang, L.; Tang, X.; Rudolph, V. Investigation of Gas Permeability in Carbon Nanotube (CNT)-Polymer Matrix Membranes via Modifying CNTs with Functional Groups/Metals and Controlling Modification Location. J. Phys. Chem. C 2011, 115, 6661-6670. [CrossRef]

41. Shen, Y.; Lua, A.C. Theoretical and experimental studies on the gas transport properties of mixed matrix membranes based on polyvinylidene fluoride. AIChE J. 2013, 59, 4715-4726. [CrossRef]

42. Kirmani, A.R.; Peng, W.; Mahfouz, R.; Amassian, A.; Losovyj, Y.; Idriss, H.; Katsiev, K. On the relation between chemical composition and optical properties of detonation nanodiamonds. Carbon 2015, 94, 79-84. [CrossRef]

43. Pichot, V.; Guerchoux, M.; Muller, O.; Guillevic, M.; Fioux, P.; Merlat, L.; Spitzer, D. Nanodiamond coating by polyethylenimine for optical limitation. Diam. Relat. Mater. 2019, 95, 55-59. [CrossRef] 
44. Petit, T.; Puskar, L. FTIR spectroscopy of nanodiamonds: Methods and interpretation. Diam. Relat. Mater. 2018, 89, 52-66. [CrossRef]

45. Murali, R.S.; Sridhar, S.; Sankarshana, T.; Ravikumar, Y.V.L. Gas Permeation Behavior of Pebax-1657 Nanocomposite Membrane Incorporated with Multiwalled Carbon Nanotubes. Ind. Eng. Chem. Res. 2010, 49, 6530-6538. [CrossRef]

46. Barnard, A.S. Diamond standard in diagnostics: Nanodiamond biolabels make their mark. Analyst 2009, 134, 1751-1764. [CrossRef]

47. Jabeen, S.; Kausar, A.; Muhammad, B.; Gul, S.; Farooq, M. A Review on Polymeric Nanocomposites of Nanodiamond, Carbon Nanotube, and Nanobifiller: Structure, Preparation and Properties. Polym. Plast. Technol. Eng. 2015, 54, 1379-1409. [CrossRef]

48. Vatanpour, V.; Salehi, E.; Sahebjamee, N.; Ashrafi, M. Novel chitosan/polyvinyl alcohol thin membrane adsorbents modified with detonation nanodiamonds: Preparation, characterization, and adsorption performance. Arab. J. Chem. 2020, 13, 1731-1740. [CrossRef] 